\title{
Understanding Players' Map Exploration Styles
}

\author{
Chen Si \\ Games Studio \\ University of Technology, Sydney \\ chen.si@student.uts.edu.au \\ Yusuf Pisan \\ Games Studio \\ University of Technology, Sydney \\ yusuf.pisan@gamesstudio.org
}

\author{
Chek Tien Tan \\ Games Studio \\ University of Technology, Sydney \\ chek@gamesstudio.org
}

\begin{abstract}
Exploration is an essential part of play in modern video games. It refers to the discovery-based activities, in which players explore mechanisms, as well as spatiality of virtual world. Exploration games and games with exploration plots are booming in gamer communities. In this paper, we focus on spatial exploration, which is central to play in role-playing games (RPG) and real time strategy (RTS) games. We investigate the game-playing behaviors of human players in exploration games, so as to discover behavior patterns and understand gamer styles. The intention is to contribute to the design and development of believable agents. We conducted an experiment where 25 participants played three types of exploration games. In-game data, think-aloud data, questionnaire responses and post-game interview data were collected to gain a deeper understanding of exploration preferences. We used thematic analysis to analyze data and mapped out four game exploration archetypes: Wanderers, Seers, Pathers and Targeters. An analysis from the four highlight aspects: strategy, reasoning, conception and hesitation, is conducted to investigate the behavioral traits of these four archetypes.
\end{abstract}

\section{CCS Concepts}

- Human-centered computing User studies $•$ Human-centered computing Empirical studies in $\mathrm{HCI} \bullet$ Software and its engineering Interactive games $\bullet$ General and reference $\sim$ Empirical studies $\bullet$ Applied computing Computer games

\section{Keywords}

Gamer typology; spatial exploration; thematic analysis; think aloud; real-time strategy games.

\section{INTRODUCTION}

Exploration is a common discovery-oriented activity that players perform in modern video games. In some games, like Journey ${ }^{1}$, the game can be purely about exploration. In other games, ranging from adventure games to first-person shooter (FPS) games, exploration is a game mechanic that players need to master in order to collect resources and advance in the game. A common type of exploration in video games is spatial exploration, which includes the discovery of locations, landmarks and specific game items, etc. Mapping of game environments is a design manifestation of the spatial exploration mechanic in games, where players have to reveal unknown environments by travelling on

\footnotetext{
${ }^{1}$ http://thatgamecompany.com/games/journey/
}

them (e.g., uncovering the fog-of-war in an RTS game). In these scenarios, players normally don't have any information about the game map at the early stage. As the player explores the game world, she builds up her knowledge of the map, making it easier for her to navigate between destinations and find game items. Oftentimes, exploration also adds to the variety of game-play, e.g., rewarding players when they uncover hidden trap doors to secret levels, unique bonus weapons, etc. For some players, diligently seeking out these surprises becomes the most enjoyable part of the game. In Super Mario Bros $3^{2}$ for instance, finding hidden flutes will allow players to almost skip the entire game.

As discussed above, exploration takes an important position in digital game playing. Human behaviors in doing exploration are, however, not understood sufficiently in scientific perspectives. Investigating how players explore in virtual game environments can contribute to better game design, e.g., how game objects are hidden and distributed around the map and the design of believable non-player characters (NPCs) that use human-like exploration techniques. As presented by Iskander and Maxim [7], human-like characters, which play with or against human-players, could essentially improve game-playing experiences. Basically, mimicking behaviors of human is a practical way to build up humanoid agents in games [8]. Prior work has sought to divide players into different groups according to their features $[2 ; 10]$. Player types have been developed that have been shown to effectively reflect behavior features of different groups of players. Correlations between these player types and psychometric types have also been presented [10]. Their player typology derivations and findings, although highly valuable to the design of believable agents and game design in general, are nevertheless lacking in their consideration of player exploration behaviors. Human behaviors of exploration in virtual worlds, which is a major component of digital games, however, have not been sufficiently studied.

The purpose of this research is hence to extract new knowledge about how players explore game virtual worlds. Our research focuses on analyzing behavior patterns and discovering new classifications of players based on exploration. We have chosen StarCraft: Brood $\mathrm{War}^{3}$ as our test-bed due to its well established API and flexibility. StarCraft is also representative of the popular RTS game genre. The StarCraft AI Competition, first hosted by the AIIDE Conference in $2010^{4}$, has also become a notable testbed for evaluation of AI agents in academia. Using thematic analysis, we analyzed data gathered from 25 participants' exploration experiences. The data revealed four player archetypes, i.e. Wanderers, Seers, Pathers and Targeters. We, then, evaluate the preferences of these archetypes on four aspects, i.e. strategy, reasoning, conception and hesitation.

\footnotetext{
${ }^{2}$ http://mario.nintendo.com/

${ }^{3} \mathrm{http} / / /$ us.blizzard.com/en-us/games/sc/

${ }^{4}$ http://eis.ucsc.edu/StarCraftAICompetition
} 
In the next section on Related Work, we review literatures about video game personalization. In the Method section, we describe the experimental environment and the data analysis approach we used. Our findings are then presented in the Results and Conclusion sections.

\section{RELATED WORK}

The broad field of research in classifying human behaviors has drawn much attention from game researchers, as understanding player personalities helps to improve player experiences when included in the game design process. Myers and McCauley [9] developed the Myers-Briggs Type Indicator (MBTI), using four bipolar axes to distinguish personality types in four dimensions. In each bipolar axis, two opposite psychological types are marked on the ends. Different permutations of these dimensions classify individuals into one of sixteen types. The MBTI is regarded as a type-based preference, which assumes that each personality is mutually exclusive. This assumption is claimed to be invalid by modern critics [5], who speak of the trait-based personality models such as the Five Factor Model (FFM) [6]. The FFM includes five personality traits (openness, conscientiousness, extraversion, agreeableness, and neuroticism). Although the FFM is widely perceived as a leading model in personality psychology, it is not practical in modeling gamers [5].

In player behavior research focused in video games, Bartle [2] presented a qualitative gamer-personality model with four types (Achiever, Explorerer, Socializer, and Killer). Yee [12] investigated and revealed five motivations (Achievement, Relationship, Immersion, Escapism and Manipulation) for players to enjoy Massively Multiplayer Online Role-playing Games (MMORPG). Yee's model also contributes to Bartle's model by building a quantitative measurement for it. It was highlighted that a limitation for both Bartle and Yee's model is their narrow focus on massively multiplayer games, which led to the development of a relatively new model - BrainHex [11] that presents seven gamer types (Achievers, Conquerors, Daredevils, Masterminds, Seekers, Socializers and Survivors). Being founded on neurobiological theories and validated with large number of participants, the BrainHex model attempts to provide a more generalizable typological model across game genres.

\section{METHOD}

Our approach involves capturing and analyzing data from participants playing three custom-designed, time-restricted game scenarios created on the StarCraft: BroodWar platform. Participants were instructed to perform a concurrent think-aloud, i.e., verbalize what they were looking at, doing and thinking during the gameplay. The think-aloud data was combined with video-cued retrospective interview post gameplay to obtain further insights into participants' experiences and thought processes. We recorded participants think-aloud and interview videos, and then used NVivo ${ }^{5}$ to transcribe and analyze the transcripts. To help contextualize our results, we will first briefly describe the StarCraft platform and game scenarios we developed.

\subsection{The StarCraft Game}

A RTS game is a game, in which making-decisions in both general strategies and specific actions takes the primary role in game playing. All RTS games require players have a high-level of situational awareness. High speed of operations is also a significant factor in determining the outcome of a round of game

\footnotetext{
${ }^{5}$ http://www.qsrinternational.com/products_nvivo.aspx
}

playing, because players perform actions in parallel. In a typical RTS game, players maneuver units to control of regions and gather resources. Resources are, then, used to either manufacture more units, including production units and combat units, or advance techniques to enhance combat and general abilities. The condition of winning a game is to eliminate the opponent base.

StarCraft: BroodWar, which was developed and released by Blizzard Entertainment in 1998, is a typical RTS game, premised on fictional interstellar wars. Players are required to select a race among three options, i.e., Protoss, Terrans, and Zerg, when starting a campaign. Each race would provide the player with different units and strategic options in general.

\subsection{Game Environment}

To collect more representative data about exploration activities, we developed three different game scenarios: the pure exploration game, the killing game and the searching game. In all the game environments, maps are covered by fog-of-war initially. Information about what the terrain looks like, and what items there are under the fog, is hence initially hidden from the players. Participants will need to explore the environment by navigating a unit with a limited perception range in the unknown environments. Areas are revealed to players when perceived by the game unit. Each game requires players to finish a specific task within a certain limited time.

- In the pure exploration game, participants are required to explore the whole map as fast as possible with a time limitation of three minutes.

- In the killing game, there are 41 enemy SCVs (a basic StarCraft unit) located on the map. It requires players to maneuver a marine to hunt the 20 of the 41 SCVs within five minutes. Since the SCVs do not move, the difficulty is in exploring the environment to discover them.

- In the searching game, participants are required to find the enemy base within four minutes. Participants were told that enemy base is located near to a mineral site, and enemy's supply depots (a basic StarCraft building) are distributed around the base area to provide them with some guidance in finding the enemy base.

The design of these three exploration games caters to two types of applications that this research can benefit. The first type is the development of autonomous exploration agents. As Francesco Angigoni [1] presented, several applications, ranging from classical map building to search and patrolling, falls within the scope of the exploration problem. Correspondingly, the pure exploration game simulates typical instances of classical map building applications, while the searching game simulates the search applications. The killing game simulates patrolling applications. The other type of applications refers to digital games with spatial exploration mechanisms. These three exploration scenarios represent a large amount of exploration mechanisms in games. For example, players might explore game environments to cumulatively acquire information for their strategic decisions. Or they may explore in order to collect items, discover eastern eggs, or seek out secret dungeons for bonus challenges.

\subsection{Participants}

Participants were recruited via undergraduate and postgraduate university mailing lists, public social networks and public areas in the university. In total, twenty-five participants ( 7 females, 18 males) signed up. After consenting to the study, participants filled 
an online pre-play survey to collect basic demographics like age, gender, gaming interests, gaming habits, and how familiar they were with RTS games and StarCraft. Participants were aged between 20 and $44(\mathrm{M}=29, \mathrm{SD}=6.01)$. Except for two participants, most have rich video game experiences. Eleven participants usually play strategy games, ten participants play first- person shooter games, seven participants play sports games, seven participants play role-playing games, four participants play simulation games, seven participants play puzzle games and three participants play social games. Of the twenty-five participants, ten indicated that they were familiar with RTS games, three claimed that they were experienced StarCraft players and four claimed that they could recognize some StarCraft maps. One participant indicated that he plays games for more than 20 hours per week, four participants between 10 to 20 hours, one participant between 5 to 10 hours, seven participants between 1 to 5 hours and twelve participants less than 1 hour per week.

\subsection{Procedure}

After participants completed the survey, the experimenter displayed a game demo to illustrate how to control and navigate game units and explained the targets that need to be achieved in each game. After that, the participants started to play the games. The playing order of the three games is generated as random. During gameplay, participants were asked to perform the concurrent think-aloud. They were encouraged to describe what strategies they made to play the game and what their instant strategic thoughts and preferences were, and to explain their behaviors to the experimenter. Prompting was also performed to encourage participants to verbalize continuously. After each game was finished, participants filled out a post-game questionnaire, in which participants are encouraged to figure out their gameplaying behavioral preferences and memories about the game. After they filled out the post-game questionnaire, a post-game interview is conducted while watching a video replay of the game they have just completed. Participants get an additional opportunity to explain what they were thinking and feeling during gameplay; in case they missed out any important thoughts during the think-aloud.

\subsection{Data Analysis}

Thematic analysis is used to analyze the experimental data, due to its flexibility to explore data in depth and formulate structures. In general, the data analysis process is grounded on the thematic analysis phases [4]. An iterative inductive approach is developed and applied, which adapts to the experimental environment and associated facilities. To be specific, it consists of four stages to forge the discovery report of human manners in exploring digital game environments.

\subsubsection{Develop Proposal Codes and Themes}

In our experiment, the data analyzer observed the entire experiment, which allowed the analysis to start at the data collection stage. During the experiment, similar strategies related to similar responses to the same questions were observed among participants. Observing all the testing and recording provided an integral view of the data. The analyzer recorded participants' think- aloud during gameplay and their responses in the postgame interview. After all the participants completed the experiments, the analyzer summarized his notes according to the topics of strategic preferences, reasoning manners and personalities into associated categories. These structured topics were used as initial draft themes for the data analysis.

\subsubsection{Data Preparation and Familiarization with} Data

For the current stage, we have collected verbal data within thinkaloud and interview, and game replay records. The game replay and verbal data is recorded synchronously. This means the analyzer could review the camera shot of either think-aloud or interviews and the game replay videos synchronously, which provides a succinct way of recovering what happened during the experiments. Raw data from audio resources is converted into textual files to meet the requirements of marking and coding in later stages of analysis. The verbal data is transcribed into textual form, along with game situation descriptions, participant behaviors and comments via Nvivo.

The analyzer, meanwhile, is familiar with the data comprehensively by repeated reading in an immersive way. It started with overall- reading and re-taking notes of contents, which are potential to be themes. Then, the analyzer read the data by searching for contents of draft themes, and responses patterns across the data.

\subsubsection{Code the Data and Extract Themes}

In this phase, we start by extracting interesting features from the data set into code. Each code refers to "the most basic segment, or element, of the raw data or information that can be assessed in a meaningful way regarding the phenomenon" [3]. The coding process is conducted on the both textual think-aloud and interview data. It mainly focuses on the data that describes the act of exploration. The game-playing video data is used as actual materials for better understanding the meanings of textual descriptions.

The analysis process begins with constructing a hierarchical preliminary theme structure on the basis of initial theme drafts generated in the first phase. Then, the analyzer encodes the data. After that, the code is collected into relevant potential themes. If there is no corresponding theme, a new theme is created and inserted into the structure. The process continues until all the data has been processed.

\subsubsection{Reviewing and Re-Construing Themes}

This phase starts with the analyzer reviewing the themes. It involves the refinement, redefinition, and reorganization of themes. Of the potential themes, some might lack support from the data, and some might be confusing due to the conflicted meanings of codes that they include. The analyzer then prunes these themes, releases codes from them, creates new themes, and categorize these codes into new themes.

After that, the analysis proceeds to reorganize themes, aiming to categorize them into common aspects of spatial exploration. This resulted in four main themes:

- strategy, which represents what strategies people made to complete tasks;

- reasoning, or how they reasoned about situations and targets;

- conception, which represents what spatial conceptions about the environments mapped in their minds;

- hesitation, their reduplicative movements in the game.

Each theme represents a common behavioral aspect that participants performed in the three exploration games. When reviewing the data sets grouped into these four themes, we discovered that participants have distinguishable preferences. Within each theme, we then embark on an iterative code-mapping process to re-organize the codes into different groups according to 
these preferences. We found four archetypes, Wanderers, Pathers, Targeters and Seers, across the four themes. Sub-themes and codes within these four themes are then regrouped into these four archetype-themes. The characteristics of these archetypes and their performances on the themes are discussed in the next section.

\section{RESULTS}

\subsection{Archetypes}

From the analysis above, four player archetypes, i.e. Wanderers, Seers, Pathers and Targeters, are revealed. In the next section, we describe each of them and give examples to illustrate how they perform in the games.

\subsubsection{Wanderers}

Regardless of the game type or immediate goals, Wanderers move about without a definite destination or purpose. Wanderers do not have high-level understanding of their location or specific plans on how to reach their next destination. Their concentration is applied exclusively in local areas and items that they instantly discover within the sight cone of the explorer. Wanderers prefer local landmarks, observing nearby terrains and regarding edges as references to navigate. A typical Wanderers quote is "I just randomly select the direction. Follow the edge of the map." (P4 Interview - Pure exploration game) This shows that he had no set targets and that he had minimal awareness of map features.

\subsubsection{Seers}

Seers aggressively expand their visual range in detecting unknown environments. The visible attribute of areas is a sensitive element for them, which leads to a clear concept of the size and location of explored places being kept in their mind. Revealing unexplored environment in both scrutinized and aggressive ways takes the priority. For example, a participant employed depth-first-like search strategy in playing the pure exploration game, aiming to perceive more areas aggressively. It is demonstrated by the statement, "I just want to do depth-first search. I just want to finish [exploring] all the far points. Because, I think, the bottom one [area] is just very close to the start point. So, it won't be a very large area. Because I have already discovered the right side [of the map] - the bottom right side. So, I think, the bottom left side [of the map] won't be a big area." (P3 - Think aloud - Pure exploration game)

\subsubsection{Pathers}

The Pather archetype is characterized by the elaborate mental maps that are created of the environment. The terrain features, such as high land, low land, ramp, bridges etc., are highlighted, perceived, slotted and grouped. Pathers recognize a map into a specific pattern group by analyzing its appearance and reasoning about its functional features. Even though, in most cases, the view of the entire environment is not available for a player, they are keeping an eye on the elements, which play important roles in making up map patterns, consistently. A pattern for the map is accumulatively constructed along with the discovery of landmarks in the terrain. The example of the archetype is extracted from interview data that "At this point, I was confused by the map, because I realized there is a layer. But I forgot. I completely forgot where the way is." (P21 - Interview - Killing game) the participant stated her confusion. When asked by the experimenter on what the 'layer' referred to, the participant answered "The high land. At the point, I realized that this high land is, somehow kind of, a bridge [linking] to different areas. So I need to climb maybe up and down to find a way out [from areas to bridge]." shows that a structured map representation is generated in her mind.

\subsubsection{Targeters}

Performing exploration in unknown spatial environments makes players easily lose track of their goals, since it is difficult to formulate explicit plans with incomplete information. The Targeter archetype, however, is explicitly goal-oriented. Motivated by winning the game, their behaviors are exhibited with tracking targets, which is underpinned by targeted tactic plans. Targeters seek landmarks, items and all other kinds of identified objects that might be hints. They make predictions about the directions of targets; verify their predictions and adjust the plans on the basis of the fresh discoveries. For example, a Targeter said (P20 - Interview - Searching game) "I just realize that I was getting closed to [the base]. Then I thought it's in the corner, to be honest. Then I thought [that I have] to go and check that corner. I thought [it] may be there, maybe in the small corner that I can't see. That's why I explored it and, OH, NO. It's not there. Then I went back. Here, I couldn't believe that I am wrong." The participant's disappointment shows her level of confidence about her prediction. When asked by the experimenter to elaborate, she responded "[The base should be] exactly between these two [mineral site]. Now, yeah, I began to find [it]. And then I saw this [supply depot]. I thought, OK, it could be something around this area [pointing to the middle black area of the map]. And then I saw a path there". It appeared that she was keeping track of hints, as well as analyzing them constantly, in order to locate the opponent's base.

\subsection{Lens Analysis}

Within the next section, we describe how the behaviors of the four archetypes differ in terms of the four behavioral aspects: strategy, reasoning, conception and hesitation.

\subsubsection{Strategy}

Different archetypes express their strategies differently based on their preferences.

Wanderers do not possess any systematic strategies. At the early stages of exploration, a Wanderer is more likely to choose a random direction to move forward. Their typical thought processes are "I have no idea" (P5 - Think aloud - Pure exploration game) and "I just randomly select a path" (P3 Interview - Searching game). Subsequently, tracking terrain features, e.g., boundaries, is a common type of behaviors. A Wanderer (P6 - Interview - Pure exploration game) described his exploration strategy as: "Since I have to find, say, all of the areas, I decided to follow the edges, because if I do, eventually I will complete [exploring] the whole [map]."

Seers keep a global view of the environment. Their general strategies are normally direction-oriented, which include an explicit sequence of exploration to cover different sections of the map. A Seer (P4 - Interview - Killing game) described his strategy as, "I found [realize] the overview map. I found I was on the top [side of the map]. So I wanted to go down [side of the map] to search another place." Additionally, this sole focus on map uncovering is also a strategic priority for Seers when considering strategies in the killing game and the searching game, where uncovering unknown terrain is not the main task. For instance, a Seer (P8 - Interview - Searching game) said, "What I am trying to do is to [go] across the whole map. Not just to find the short way."

Pathers take advantage of the structure in maps. They normally define enclosing areas where they have already explored, and prefer to uncover a region completely before they move to explore 
another area, e.g., when a Pather was asked why he went a certain direction, he replied "because I thought if I go down [side to the area], I don't have to like go back." (P19 - Interview - Pure exploration game). Another behavior identified was that they are actively looking for terrain connections among different parts of maps, e.g., "I found there is no way to leave this area. So I have to go back. I saw a bridge" (P4 - Think aloud - Pure exploration game); "I just want to search a connection between this part and another part." (P2 - Think aloud - Pure exploration game)

\subsubsection{Reasoning}

During the process of exploration, players will reason about unexplored areas, paths and targets based on what they have already discovered, and different archetypes do it in a different manner.

Wanderers normally choose where to go within the limited local view of the map base on random guesses. For example, we observed a Wanderer participant who navigated his unit towards the path to the right side of the map. When asked why he didn't go to the path that leads downwards, he said, "I, sort of, assume that the cliff was there [downward direction]." (P6 - Interview Searching game)

Seers prefer navigational options that can result in larger visible regions. For example, a Seer participant explained his choices as "There is also much area to explore. So I move down [side of the map] for efficiency. So I start going south [down side of the map]. There is no more area to the east [right side of the map]." (P11 Interview - Killing game)

Pathers keep a structured representation of the map and a clear prioritized sequence of where to go in strategic order. For example, we observed a Pather participant choosing a certain path, as opposed to an alternative path, to an area which she had partially uncovered. When asked about her reasoning, she said, "Because I saw it [the area] from the other side. I couldn't access it. So I go to that [area] first, clean that area." (P19 - Interview Pure exploration game)

Targeters reason in a way that is consistent with their goaloriented preferences, i.e., finding key objects of interest such as the opponent's base. Their reasoning process is anchored on the accumulation of hints from map features in order to predict locations of targets within unexplored areas. For example, when asked about the reasoning on why a Targeter participant chose to search in the high platform (which led him to find the opponent base eventually), he replied, "Because, at that point, I think that base is really close. The first time I saw that one ... At the first time I saw this building [supply depot], and after a few seconds I saw another one, I think it's because these buildings are around the enemy [opponent] base, so I think maybe it's in the middle. So I go up.” (P13 - Interview - Searching game)

The Pather archetype is characterized by the elaborate mental maps that are created of the environment. The terrain features, such as high land, low land, ramp, bridges etc., are highlighted, perceived, slotted and grouped. Pathers recognize a map into a specific pattern group by analyzing its appearance and reasoning about its functional features. Even though, in most cases, the view of the entire environment is not available for a player, they are keeping an eye on the elements, which play important roles in making up map patterns, consistently. A pattern for the map is accumulatively constructed along with the discovery of landmarks in the terrain. The example of the archetype is extracted from interview data that "At this point, I was confused by the map, because I realized there is a layer. But I forgot. I completely forgot where the way is." (P21 - Interview - Killing game) the participant stated her confusion. When asked by the experimenter on what the 'layer' referred to, the participant answered "The high land. At the point, I realized that this high land is, somehow kind of, a bridge [linking] to different areas. So I need to climb maybe up and down to find a way out [from areas to bridge]." shows that a structured map representation is generated in her mind.

\subsubsection{Conception}

Conception here refers to how a player constructs a cognitive representation of the game map, which varies among different archetypes.

Seers apply a direction-based approach in structuring the cognitive map, e.g., segregating a map into the top-left, top-right, bottom-left and bottom-right parts. An example of this type of segregation can be seen in a Seer participant's thought process: "My strategy is just to walk from left [side of the map], and to the right [side of the map]. And up [side of the map] and from right [side of the map] to left [side of the map].” (P25 - Interview Pure exploration game)

Pathers cumulatively maintain a structured cognitive map based on each new acquired knowledge of the environment. They normally have a pre-conceived notion of the layout of the environment, and cumulatively construct the cognitive map by incorporating the gradually acquired spatial knowledge. Topological-map-like structures normally exists in Pathers' minds. For instance, the experimenter made an assumption about a Pather participant's behavior: "It seems that when you have explored one part, you prefer to carefully explore all the path in that area, instead of just roughly looking around and go outside. Am I right?" She responded, "No, you said outside, but I don't know outside. I expect all the areas are connecting. So I was finding a path to go out." (P18 - Interview - Pure exploration game)

\subsubsection{Hesitation}

A common behavior observed is travelling back and forth in explored areas. The reasons, however, come with rich possibilities, due to the diverse situation. We define and classify this kind of behaviors as hesitation. Different archetypes are driven by different motivations to perform hesitations.

Wanderers hesitate for two reasons: lacking of specific strategies and being unfamiliar with environments. The combination of these two reasons is especially apparent in the killing game. "Why did you keep on moving forward and backward within the areas you have explored?" asked the experimenter. "Because I am sure that I have been here at the first time, and I regarded the fly thing as an enemy [opponent unit]. But I was wrong. I think I didn't have a CLEAR VISION of the map. I didn't have some theories about how to explore the new enemies [opponent units]. So, I just moved back and forward." the participant (P2 - Interview Killing game) said.

Pathers' hesitated behaviors are generated from their hesitations in ordering the sequence of visiting points next. For instance, one participant (P16 - Interview - Pure exploration game) explained his hesitated movements saying, "I didn't take the other view - the right side view. I missed one connecting path, so I return there. So I thought maybe if I come back to the same place. Then I would find a way to go to the right [side of the map]. Then I went to up [side of the map] again, I can't find way to right. I spent some time near here". When we look at the game replay, we found that 
he had explored the left part of the map, and was attempting to find a connection to explore the right side.

Targeters' hesitation in exploring environments with behaviors of walking back and forth caused by their purpose of finding out all hidden objects. A typical example that a participant (P4 Interview - Searching game) gave up moving on his current path and moving back to explore repeatedly. The experimenter asked, "Why did you go back?" "I didn't find the base [that is] around this supply depot. And I think I missed some places in the left [side of the map]. So [I] came back." he answered.

\section{DISSCUSSION}

Even though the four archetypes we discovered were based on our unique study environments aimed at investigating exploration behaviors, it is interesting to note that they share similarities to

other more general gamer types devised in prior research [10]. The relations between our four archetypes and Nacke's types are described below:

- The Wanderer is related to Nacke's Seeker type. Similarities include the preference of seeking instant and easy enjoyment from the environment. The Wanderer is also partially related to Nacke's Survivor type, since negative emotions with the sense of fear would motivate players to focus exclusive on their immediate localities and not see or plan for the broader exploration task.

- The Seer can be regarded as an aggressive version of Nacke's Seeker type. Unlike the Wanderer type, in which players' behaviors can be associated with a sense of lost, the Seer type tends to be more aggressive and risk taking. This characteristic also coincides with the characteristics of Nacke's Daredevil type.

- The Pather is related to Nacke's Seeker type as well, but with the addition of maintaining a structured mental map. It is also related to Nacke's Mastermind type which is described as being curious about the structure of virtual environments.

- The Targeter is related to Nacke's Achiever type, as both are centered on goal-oriented behaviors. The Targeter is also related to Nacke's Mastermind type, because Targeters also exhibit strong qualities in devising effective strategies for achieving their targets, similar to the characteristics described for Masterminds

\section{CONCLUSION}

We investigated how people explore virtual environments by using thematic analysis to extract themes from both think-aloud and retrospective interview recordings. Through custom-designed exploration-oriented games we developed, we found four exploration archetypes of players: Wanderers, Pathers, Targeters and Seers, according to their in-game behaviors and how they describe their actions. We also investigated how the gamer archetypes perform in the four aspects: strategy, reasoning, conception and hesitation. Our results forge four archetypes for players in spatial exploration games, which could be the basis of gamer typology research in this area. Further studies about generating quantitative prove of behavior differences or of existence of actual clusters of behaviors are our future work. Additionally, since some researches put efforts to mimic human strategies in developing autonomous exploration strategies, such as wall-following and frontier-based, discovering the quantitative correlation between player types and human-behavior-simulated strategies can be an interesting research spot.

\section{ACKNOWLEDGE}

We greatly appreciate Leila Alem for her advises in designing the experiments. This research is supported by the University of Technology, Sydney and the China Scholarship Council.

\section{REFERENCES}

[1] Amigoni, F., Basilico, N., and Quattrini Li, A., 2014. Moving From 'How to go There?' to 'Where to go?': Towards Increased Autonomy of Mobile Robots. In New Trends in Medical and Service Robots, A. RODIĆ, D. PISLA and H. BLEULER Eds. Springer International Publishing, 345-356. DOI= http://dx.doi.org/10.1007/978-3319-05431-5_23.

[2] Bartle, R., 1996. Hearts, clubs, diamonds, spades: Players who suit MUDs. Journal of MUD research 1, 1, 19.

[3] Boyatzis, R.E., 1998. Transforming qualitative information: Thematic analysis and code development. Sage.

[4] Braun, V. and Clarke, V., 2006. Using thematic analysis in psychology. Qualitative Research in Psychology 3, 2 (2006/01/01), 77-101.

DOI=http://dx.doi.org/10.1191/1478088706qp063oa.

[5] Chris, B., Rebecca, L., and Lennart, N., 2011. Player Typology in Theory and Practice. In Proceedings of the DiGRA - Proceedings of the 2011 DiGRA International Conference: Think Design Play (2011), DiGRA/Utrecht School of the Arts.

[6] Goldberg, L.R., 1993. The structure of phenotypic personality traits. American Psychologist 48, 1, 26-34. DOI= http://dx.doi.org/10.1037/0003-066X.48.1.26.

[7] Iskander, U. and Maxim, M., 2012. Believable and Effective AI Agents in Virtual Worlds: Current State and Future Perspectives. International Journal of Gaming and ComputerMediated Simulations (IJGCMS) 4, 2, 37-59. DOI= http://dx.doi.org/10.4018/jgcms.2012040103.

[8] Karpov, I., Schrum, J., and Miikkulainen, R., 2012. Believable Bot Navigation via Playback of Human Traces. In Believable Bots, P. Hingston Ed. Springer Berlin Heidelberg, 151-170. DOI= http://dx.doi.org/10.1007/978-3-642-323232_6.

[9] Myers, I.B., Mccaulley, M.H., and Most, R., 1985. Manual, a guide to the development and use of the Myers-Briggs type indicator. Consulting Psychologists Press.

[10] Nacke, L., Bateman, C., and Mandryk, R., 2011. BrainHex: Preliminary Results from a Neurobiological Gamer Typology Survey. In Entertainment Computing - ICEC 2011, J. Anacleto, S. Fels, N. Graham, B. Kapralos, M. Saif El-Nasr and K. Stanley Eds. Springer Berlin Heidelberg, 288-293. DOI= http://dx.doi.org/10.1007/978-3-642-24500-8_31.

[11] Nacke, L.E., Bateman, C., and Mandryk, R.L., 2014. BrainHex: A neurobiological gamer typology survey. Entertainment Computing 5, 1, 55-62.

DOI=http://dx.doi.org/http://dx.doi.org/10.1016/j.entcom.201 3.06. 002 .

[12] Yee, N., 2006. The Demographics, Motivations, and Derived Experiences of Users of Massively Multi-User Online Graphical Environments. Presence 15, 3, 309-329. DOI= http://dx.doi.org/10.1162/pres.15.3.309 